\title{
Placental Mesenchymal Dysplasia: Ultrasound Characteristics and Diagnostic Pitfalls
}

\section{Introduction}

Placental mesenchymal dysplasia (PMD) is a rare, benign developmental anomaly with a reported prevalence of $0.02 \%$ (Arizawa and Nakayama, 2002). It is characterized by placentomegaly with multiple cystic lesions of the stem villi and vascular anomalies (Pawoo and Heller, 2014). Early detection of PMD has been described during routine prenatal ultrasound (Vaisbuch et al., 2009). The sonographic characteristics of PMD include increased placental thickness and multiple cystic areas within the placenta with either an absence of blood flow or with low venous Doppler signals (Vaisbuch et al., 2009). The differential diagnosis of multicystic placental lesions with the presence of a live fetus include partial molar pregnancy, multiple hematomas, chorioangioma Beckwith-Wiedemann syndrome and PMD. Chorioangiomas are well circumscribed masses within the placenta and they are characterized by the presence of a single feeding vessel with the same pulse rate as the umbilical cord (Zalel et al., 2002). Invasive prenatal testing is required for the exclusion of partial molar pregnancy and Beckwith-Wiedemann Syndrome (Vaisbuch et al., 2009). Definitive diagnosis of PMD is based on the pathologic examination of the placenta. Histology reveals aneurysm or dilated blood vessels that may be thrombosed. The stem villi are edematous and enlarged with thick-walled vessels, without trophoblastic proliferation (Pawoo and Heller, 2014). This case report highlights the significance of the early detection of PMD, illustrates the pitfalls in differential diagnosis and provides valuable insights regarding PMD management in a clinical setting.

\section{Case Presentation}

We present the case of a 30-year-old primigravida, who visited the outpatient clinic of "Alexandra" Maternity Hospital in Athens, Greece. Her past medical history was uneventful. The first-trimester ultrasound scan performed at 13 weeks of gestation revealed increased placental thickness for the gestational age (above the $95^{\text {th }}$ percentile) (Karthikeyan et al., 2012). Closer examination of the placenta revealed nu-

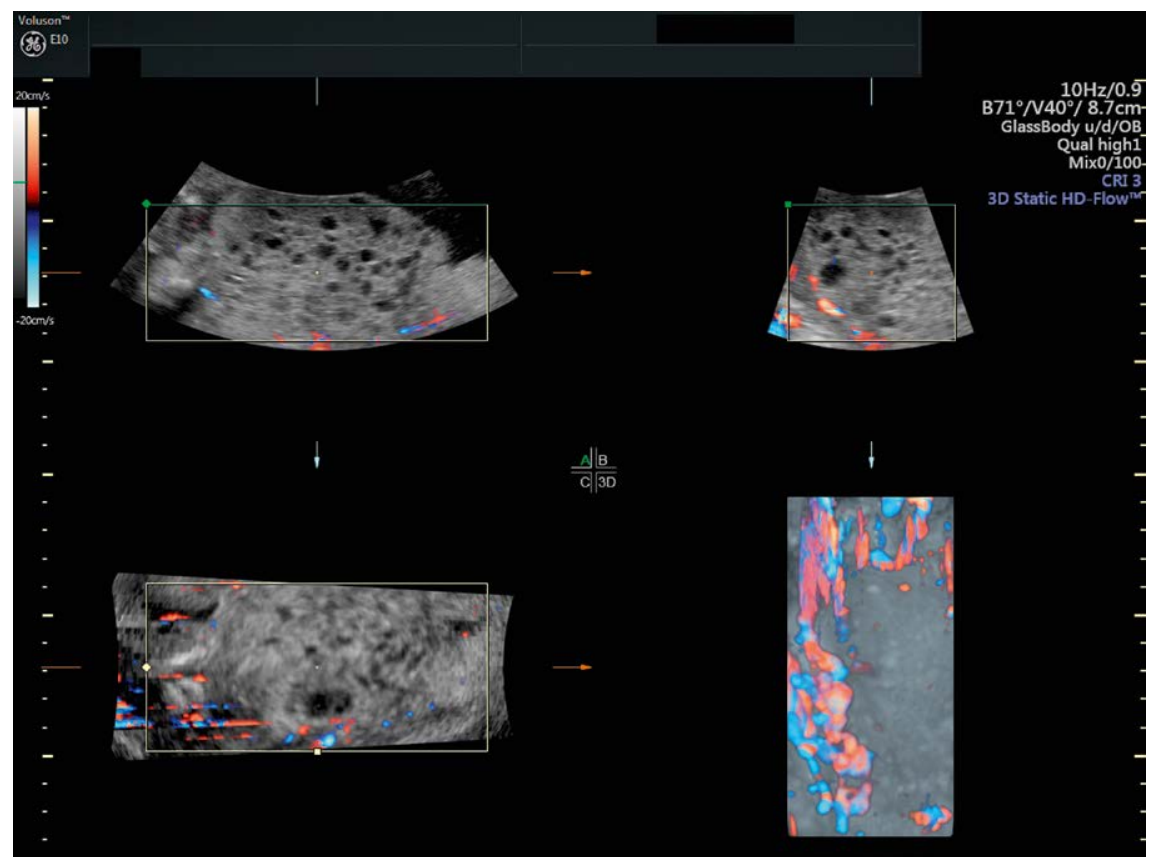

- Fig. 1 Placental mesenchymal dysplasia at 21 weeksof gestation. The placental area with the multiple cystic lesions measured $10.53 \times 4.66 \times 7.35 \mathrm{~cm}$. Color Doppler revealed the absence of vascular flow.

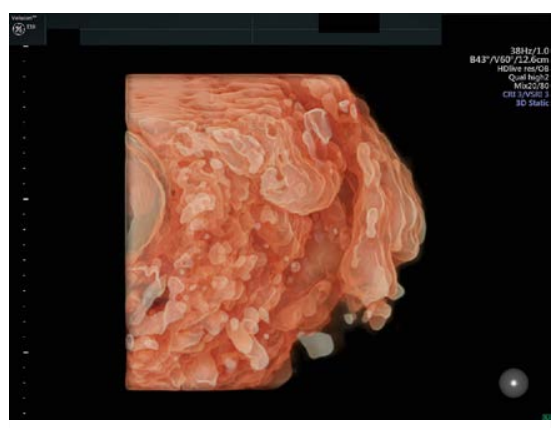

Fig. 2 3D ultrasonography revealed multiple small vesicles in the uterine cavity.

merous anechoic cystic lesions. Doppler examination did not reveal the presence of blood flow within the placental lesions. The fetal anatomy was normal, the nuchal translucency was $1.7 \mathrm{~mm}$ and the amniotic fluid was within normal range. The appearance of the placenta on ultrasound combined with the presence of a live fetus could be attributed to partial molar pregnancy, mesenchymal placental dysplasia, Beckwith Wiedemann syndrome or multiple hematomas. The free $\beta$ hCG was $33.77 \mathrm{IU} / \mathrm{L}(0.83$
MoM) and the serum PAPP-A was 3.790IU/L (1.587 MoM). Genetic counseling was advised, followed by amniocentesis, arraybased comparative genomic hybridization (aCGH) and methylation-specific multiple ligation probe amplification analysis (MSMLPA) for epigenetic changes associated with Beckwith Wiedemann syndrome. aCGH showed a normal female karyotype and MS-MLPA was negative for Beckwith Wiedemann Syndrome. The second-trimester anomaly scan was normal, with the exception of marginal bilateral renal pelvis dilation (5.3 and $5.5 \mathrm{~mm}$, respectively). The placental area with the multiple cystic lesions was $10.53 \times 4.66 \times 7.35 \mathrm{~cm}$ and color Doppler still revealed no vascular flow (\$ Fig. 1). 3D ultrasonography revealed multiple small vesicles in the uterine cavity, originating from the placenta ( $\triangleright$ Fig. 2 ). During the routine ultrasound scan in the $32^{\text {nd }}$ week of gestation, growth arrest was detected. The estimated fetal weight, calculated via the Hadlock formula, dropped from the $42^{\text {nd }}$ to the $15^{\text {th }}$ percentile. The patient was admitted to the high-risk pregnancy unit and induction of labor was deci- 


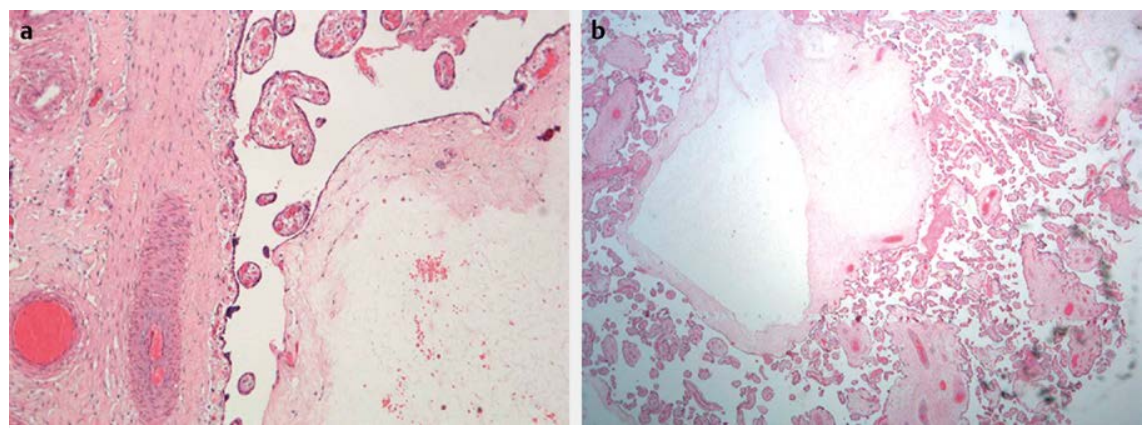

- Fig. 3 (a b) Histology revealed markedly edematous stem villi, thick-walled vessels and a lack of trophoblastic proliferation.

ded at 36 weeks of gestation. The woman gave birth to a healthy girl that weighed $2210 \mathrm{gr}$ and had an Apgar score of 9 in the first minute and 10 in the $5^{\text {th }}$. The placenta weighed $720 \mathrm{gr}$, which is over the normal limit for the gestational age. Pathology examination of the placenta confirmed the diagnosis of placental mesenchymal dysplasia (॰ Fig. 3).

\section{Discussion}

Despite the rarity of MSD, the presence of a normal appearing fetus on three-dimensional sonography along with a thick placenta with multiple cystic lesions with low or absent vascular flow on color Doppler evaluation point in the direction of PMD.

When PMD is included in the differential diagnosis, it is essential to rule out partial molar pregnancy, since the management varies greatly. Hence, invasive prenatal testing (chorionic villus sampling or amniocentesis) is required to evaluate the fetal karyotype (Vaisbuch et al., 2009). Further management includes MS-MLPA analysis to exclude Beckwith-Wiedemann Syndrome.

Antenatal genetic counselling is essential as placental mesenchymal dysplasia has been associated with diverse perinatal outcomes, ranging from uneventful delivery of a healthy neonate to significant maternal and infant morbidity. Both parents and clinicians must keep in mind that PMD has been associated with vaginal bleeding, preterm delivery (33\%), fetal anomalies (such as liver cysts and vascular malformations), fetal growth restriction (33\%), stillbirth (13\%) and Beckwith-Wiedemann syndrome (omphalocele, macroglossia, macrosomia, organomegaly) (Matsui et al., 2003; Nayeri et al., 2013). Incidental discovery of PMD in pregnancies with normal perinatal outcomes has also been described (9\%) (Nayeri et al., 2013).

Pregnancies with suspicious sonographic findings should be monitored closely, due to the increased risk of adverse perinatal outcomes. In the third trimester intense supervision along with consecutive growth scans and evaluation of fetal wellness are recommended to ensure the delivery of a healthy infant. After delivery the placenta should be sent for pathological assessment to verify PMD (Nayeri et al., 2013).

\section{Conflict of Interest}

The authors declare that they have no conflict of interest.

\section{Affiliations}

Alexandros Psarris ${ }^{1}$ D, Michail Sindos 2 , Ploutarchos Kourtis ${ }^{3}$, Andreas Pampanos ${ }^{4}$, Panagiotis Antsaklis ${ }^{1}$, Marianna Theodo$\mathrm{ra}^{1}$, Maria Eleni Chondrogianni ${ }^{1}$, Georgios Morphopoulos ${ }^{5}$, Dimitrios Loutradis ${ }^{2}$, Georgios Daskalakis²
Affiliations

1 First Department of Obstetrics and Gynecology, National and Kapodistrian University of Athens, Athens, Greece

2 1st Department of Obstetrics and Gynecology, Athens University, Athens , Greece

3 Fetal Maternal Medicine, Private Practice, Athens, Greece

4 Genetics Department, "Alexandra" Maternity Hospital, National and Kapodistrian University of Athens, Athens, Greece

5 Pathology Department, "Alexandra" Maternity Hospital, National and Kapodistrian University of Athens, Athens, Greece

\section{Correspondence}

\author{
Dr. Alexandros Psarris \\ First Department of Obstetrics and \\ Gynecology, National and Kapodistrian \\ University of Athens \\ V. Sofias 80 and Lourou Street \\ 11528 Athens \\ Greece \\ Tel.: 6979232977 \\ psarris.alexandros@gmail.com
}

\section{Bibliography}

DOI https://doi.org/10.1055/a-1180-9571

Published online: 2020

Ultrasound Int Open 2020; 6: E2-E3

(c) Georg Thieme Verlag KG Stuttgart · New York

ISSN 2199-7152

\section{(ㄷ) (1) $(8)$}

